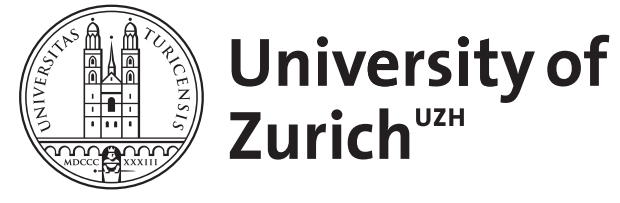

\title{
Sulla und der Kampf um das Volkstribunat
}

\author{
Thommen, Lukas
}

\begin{abstract}
L. Cornelius Sulla ging sowohl als Konsul des Jahres 88 v. Chr. als auch als Diktator im Jahre 81 v. Chr. gegen das Volkstribunat vor. Umstritten ist dabei schon lange, welche tribunizischen Kompetenzen er genau beschnitt. Der Aufsatz geht zunächst den betreffenden Beschränkungen nach und setzt sie zu den bekannten Handlungen der Volkstribunen und dem Charakter des Tribunats in vorsullanischer Zeit in Beziehung. Daraufhin wird der Kampf um die Restituierung der vollen tribunizischen Rechte in den 70er Jahren v. Chr. analysiert und gefragt, wer damit welche Interessen verfolgte. Das Volkstribunat erweist sich dabei als traditionsreiche Institution, die weniger vom Volk (populus/plebs) selbst als von führenden Kreisen sowohl für eigenständige Politik als auch für einen generellen Ausgleich des staatlichen Systems gestützt wurde. Summary: During both his consulship of 88 B.C. and his later dictatorship L. Cornelius Sulla took steps against the tribunate of the plebs, but it has long been controversial which tribunician powers exactly he targeted. This paper first examines these restrictions in connection with the known actions of the tribunes of the plebs and the character of the tribunate in pre-Sullan times. Next, I turn to the fight for the restitution of the full tribunician rights during the seventies B.C., asking who pursued which interests in this political struggle. The tribunate of the plebs turns out to be a traditional institution which was supported less by the people (populus/plebs) than by leading men for independent political purposes as well as for a general balance of the constitution.
\end{abstract}

DOI: https://doi.org/10.1515/klio-2017-0037

Posted at the Zurich Open Repository and Archive, University of Zurich

ZORA URL: https://doi.org/10.5167/uzh-148741

Journal Article

Originally published at:

Thommen, Lukas (2018). Sulla und der Kampf um das Volkstribunat. Klio. Beiträge zur Alten Geschichte, 99(2):545-565.

DOI: https://doi.org/10.1515/klio-2017-0037 


\section{Lukas Thommen*}

\section{Sulla und der Kampf um das Volkstribunat}

https://doi.org/10.1515/klio-2017-0037

Summary: During both his consulship of 88 B.C. and his later dictatorship L. Cornelius Sulla took steps against the tribunate of the plebs, but it has long been controversial which tribunician powers exactly he targeted. This paper first examines these restrictions in connection with the known actions of the tribunes of the plebs and the character of the tribunate in pre-Sullan times. Next, I turn to the fight for the restitution of the full tribunician rights during the seventies B.C., asking who pursued which interests in this political struggle. The tribunate of the plebs turns out to be a traditional institution which was supported less by the people (populus/plebs) than by leading men for independent political purposes as well as for a general balance of the constitution.

Zusammenfassung: L. Cornelius Sulla ging sowohl als Konsul des Jahres $88 \mathrm{v}$. Chr. als auch als Diktator im Jahre $81 \mathrm{v}$. Chr. gegen das Volkstribunat vor. Umstritten ist dabei schon lange, welche tribunizischen Kompetenzen er genau beschnitt. Der Aufsatz geht zunächst den betreffenden Beschränkungen nach und setzt sie zu den bekannten Handlungen der Volkstribunen und dem Charakter des Tribunats in vorsullanischer Zeit in Beziehung. Daraufhin wird der Kampf um die Restituierung der vollen tribunizischen Rechte in den 70er Jahren v. Chr. analysiert und gefragt, wer damit welche Interessen verfolgte. Das Volkstribunat erweist sich dabei als traditionsreiche Institution, die weniger vom Volk (populus/plebs) selbst als von führenden Kreisen sowohl für eigenständige Politik als auch für einen generellen Ausgleich des staatlichen Systems gestützt wurde.

Keywords: Sulla, Volkstribunat, C. Licinius Macer, Römische Republik, Verfassung

Das Volkstribunat als Errungenschaft der Plebs im Ständekampf der frühen Republik durchlief eine bewegte Geschichte. Nachdem es in der mittleren Republik weitgehend in das politische System und in die normale Beamtenlaufbahn integriert war, wurde es seit den Gracchen (133 und 123/2 v. Chr.) wieder vermehrt als Mittel der Opposition und selbständiger bzw. popularer Politik benutzt, mit

*Kontakt: Lukas Thommen, Basel, E-Mail: Lukas.Thommen@unibas.ch 
dem die Mehrheit im Senat umgangen werden konnte. ${ }^{1}$ Dies gab dann auch den Anlass dazu, dass traditionelle bzw. optimatische Kreise gegen die Institution einschritten und somit versuchten, die hergebrachte Senatsherrschaft wieder zu festigen.

Der Patrizier L. Cornelius Sulla, der sich zuletzt im Bundesgenossenkrieg (9188 v. Chr.) militärisch bewährt hatte, wollte nach der Integration der Italiker in den Bürgerverband zunächst als Konsul des Jahres 88 v. Chr., dann als Diktator im Jahre 81 v. Chr. für neue politische Stabilität sorgen und dabei die Zusammensetzung des Senats und der Gerichte, aber auch die Ämterlaufbahn neu ordnen. Dabei schritt er jeweils nach einem militärischen Marsch auf Rom auch gegen das Volkstribunat ein, dessen Kompetenzen er beschnitt. ${ }^{2}$ Einen persönlichen Anlass hatte der Volkstribun P. Sulpicius (Rufus) gegeben, der im Jahre 88 v. Chr. das für Sulla vorgesehene Kommando im Krieg gegen Mithridates VI. von Pontos auf Pompeius übertragen und weitere unliebsame Gesetze verabschieden lassen hatte. ${ }^{3}$ Sulla verfolgte jedoch weitergehende Ziele.

Im Folgenden soll zunächst ein Überblick über die einzelnen Beschränkungen gegeben werden, wobei zugleich die Auswirkungen dieser Maßnahmen auf den Charakter des Volkstribunats in den 70er Jahren v. Chr. zu analysieren sind. Anschließend wird der Kampf um die Wiederherstellung der vollen tribunizischen Rechte genauer untersucht. Dabei ist zu fragen, welche Kreise zu welchem Zweck die uneingeschränkte tribunicia potestas forderten und wieso die sullanischen Einschränkungen auf Dauer politisch untragbar waren.

\section{Die Beschränkung des Volkstribunats durch Sulla}

Gemäß Appian (civ. 1,59,266) setzte Sulla bereits im Jahre 88 v. Chr. zusammen mit seinem Kollegen im Konsulat, Q. Pompeius Rufus, dem Volkstribunat einige gesetzliche Schranken. Er verfügte, dass nichts ohne Vorberatung im Senat vor das Volk ( $\delta \tilde{\eta} \mu \circ \varsigma)$ gebracht werden durfte. Eine zweite Anordnung lautete nach Appian: „auch sollten die Abstimmungen nicht nach Tribus, sondern - eine

1 Zur mittleren Republik Bleicken (1959); Williams (2004); zur späten Republik Thommen (1989); zu den Popularen Meier (1965); Martin (1965); Robb (2010).

2 Zur Beurteilung dieser Maßnahmen vgl. nach Lengle (1899) 10 ff. in neuerer Zeit u. a. Wittmann (1984) 568 f. 574 ff.; Hantos (1988) 74 ff.; Kunkel - Wittmann (1995) 654 ff.; Keaveney (2005) 141.

3 Broughton (1952) 41; Thommen (1989) 79 ff. 95. 97; Kunkel - Wittmann (1995) 655; Lovano (2002) $19 \mathrm{ff}$. 


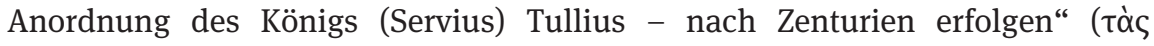

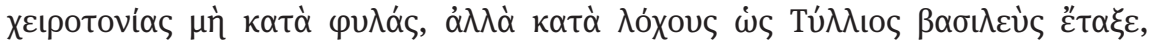

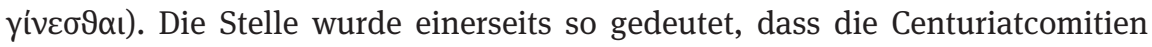
wieder nach der alten servianischen Centurienordnung organisiert wurden - sei es nach dem Modell vor der Centurienreform des 3. Jh. v. Chr. ${ }^{4}$ oder durch die Beseitigung einer durchmischten, nicht streng nach den Besitzklassen abgestuften Abstimmung, wie sie der Volkstribun C. Sempronius Gracchus im Jahre $122 \mathrm{v}$. Chr. vorgesehen hatte. ${ }^{5}$ Andererseits wurde auch mit einer Aufhebung bzw. Beschränkung der Tributcomitien gerechnet.

Eine Wiederherstellung der alten servianischen Ordnung wurde schon von Ed. Meyer 1898 verworfen, der vielmehr annahm, dass den Tribus generell die Befugnis zur Gesetzgebung genommen wurde. ${ }^{6}$ Eine Reform der Centurienordnung nach gracchischem Muster ist schon deshalb unwahrscheinlich, weil solche Pläne wohl nie in die Tat umgesetzt worden waren. Zudem konnten die Centurien von den Volkstribunen bei Gesetzesvorlagen gar nicht beigezogen werden, und andere Magistrate (Konsuln und Praetoren) hatten die Centuriatcomitien in vorsullanischer Zeit nicht ohne Einverständnis des Senats eingesetzt. ${ }^{7}$ Daher hatte Sulla keinen Grund, die Centurien neu zu organisieren, sondern sie vielmehr als grundlegende Organisation der Bürgerschaft wieder ins Zentrum zu stellen, wie das schon Servius Tullius vorgesehen hatte. ${ }^{8}$

Grundsätzlich ist davon auszugehen, dass die Tributcomitien bestehen blieben. ${ }^{9}$ Dies ist insbesondere wegen der Wahl der niederen Magistrate und in Analogie zu dem Sachverhalt nach den Beschränkungen des Jahres 81 v. Chr. wahrscheinlich. ${ }^{10} \mathrm{Ob}$ den Volkstribunen dabei Abstimmungsbefugnisse für Gesetzesvorlagen blieben, ist eine andere Frage. Livius (per. 89) berichtet zum Jahre 81 v. Chr., dass Sulla den Volkstribunen das Gesetzgebungsrecht nahm. Demgegenüber bestand die Absicht im Jahre 88 v. Chr. gemäß Appian nur darin, dass kein Gesetz, ehe es dem Senat vorgelegen hatte, an das Volk ( $\pi \lambda \tilde{\eta} \vartheta$ o $)$ gebracht

4 Herzog (1884) 502; Mommsen (1887/3) 270 f.; Lengle (1899) 10 f.; Lengle (1937) 2484 f.; Gabba (1967) 171 ff.; Gabba (1976) 135 f.; Seager (1994) 172.

5 Valgiglio (1956) 22f.; Nicolet (1959) $216 \mathrm{f}$. bezieht die Änderung nur auf die Wahlen; vgl. auch Meyer (1975) 319. 535 Anm. 22 und die ausführliche Diskussion bei Laffi (1967) 207 ff., bes. Anm. 96 f. u. 102.

6 Meyer (1898); vgl. auch Niccolini (1932) 146 f.; Meier (1997) 223 Anm. 106; Heftner (2006) 145. 7 Dies ist dann erst von Caesar im Jahre 59 v. Chr. bekannt (Broughton [1952] 187 f.; Thommen [1989] 145).

8 Vgl. die entsprechende Erklärung des Appian-Textes bei Keaveney (1983) 72 Anm. 87.

9 Lengle (1937) 2485; De Martino (1973) 68; Fabbrini (1973) 806 Anm. 3; Hantos (1988) 74. 10 S. unten S. 549. 
und auch nicht von den Armen und Frechsten entschieden werde. ${ }^{11}$ Mit dieser Bestimmung war im Prinzip das Ziel, die tribunizische Gesetzestätigkeit unter Kontrolle zu bekommen, erreicht, und ein generelles Gesetzesverbot für die Tributcomitien überflüssig. Sullas Maßnahmen zielten vielmehr darauf ab, die Möglichkeiten der Tribusversammlung einzuschränken und damit die nach Vermögen gegliederten Centuriatcomitien auch als Gesetzgebungsorgan aufzuwerten.

Schließlich erwähnt Appian (civ. 1,59,267) zum Jahre 88 v. Chr., dass neben den zwei angeführten Gesetzen noch viele andere Regelungen über die Gewalt

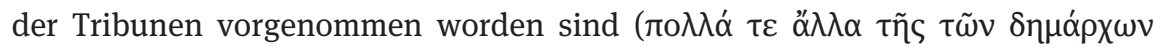

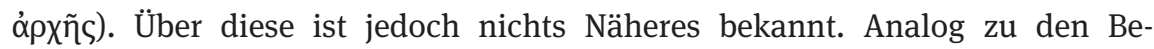
schränkungen vom Jahre 81 v. Chr. könnten sie sich gegen die Volksgerichtsbarkeit, gewisse Arten der Interzession und die spätere Laufbahn der Volkstribunen gerichtet haben. ${ }^{12}$ Einige Forscher sind davon ausgegangen, dass in der Notiz Appians zum Jahre 88 v. Chr. Regelungen des Jahres 81 v. Chr. vorweggenommen worden sind und einzig die Bestimmung über die Gesetzesvorberatung in dieses Jahr fällt, ${ }^{13}$ wofür es jedoch nur unsichere Anhaltspunkte gibt.

Klar ist, dass die erlassenen Gesetze nur bis zum nächsten Jahr in Kraft waren, da L. Cornelius Cinna als popular ausgerichteter Konsul des Jahres 87 v. Chr. nach dem Abgang Sullas in den Osten dessen Verordnungen wieder aufhob. ${ }^{14}$ Die Volkstribunen hatten aber schon Anfang des Jahres von der Interzession Gebrauch gemacht, als Cinna die Neubürger - anders als seine Gegner - auf alle Tribus verteilen wollte, ${ }^{15}$ und legten später den Tributcomitien ein Gesetz über die Rückführung des Marius und der mit ihm Verbannten vor, ${ }^{16}$ so dass das Tribunat bereits wieder von mehreren Seiten nutzbar gemacht wurde. Dass die Volkstribunen in der Folge bis zum Jahre $81 \mathrm{v}$. Chr. wieder im vollen Genuss ihrer alten Rechte waren, belegen mehrere Volksgerichte ${ }^{17}$ und Gesetzesanträge wie das Kolonisationsprojekt des M. Iunius Brutus ( 83 v. Chr. $)^{18}$ sowie die unge-

11 Lengle (1899) 10 bzw. (1937) 2484 f. meint, dass sich das Verbot eines Antrages ohne Vorbera-

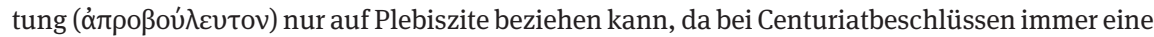
Vorberatung vorauszusetzen ist (vgl. die lex Publilia vom Jahre 339 v. Chr.; Rotondi [1912] 226f.; Siber [1952] 229f.); die Bezeichnung $\pi \lambda \tilde{\eta}$ Oos für das Volk bezieht er wie schon Mommsen (1887/3) 158 Anm. 2 auf die Plebs (Lengle [1899] 10); vgl. dagegen Meyer (1898) 653 Anm. 2.

12 S. unten S. $550 \mathrm{ff}$.

13 Niccolini (1932) 148; Schur (1942) 134 Anm. 1. 196 Anm. 2; Nicolet (1959) 216 f.; Katz (1975) 117.

14 App. civ. 1,73,339.

15 App. civ. 1,64,290; Thommen (1989) 231; Lovano (2002) $32 \mathrm{f}$.

16 Plut. Mar. 43; App. civ. 1,70,324; Thommen (1989) 125; Lovano (2002) 45.

17 Thommen (1989) 152, $156 \mathrm{f}$.

18 Rotondi (1912) 347 f.; Thommen (1989) 49 f. 54. 
hinderte Ämterlaufban des M. Marius Gratidianus (87 v. Chr.), der in den Jahren $85 / 4$ v. Chr. Praetor wurde. ${ }^{19}$

Nach seinem zweiten Marsch auf Rom konnte Sulla als Diktator im Jahre $81 \mathrm{v}$. Chr. nochmals neue, umfassende Reformen durchsetzen. ${ }^{20}$ Wesentliches Ziel seiner Maßnahmen war wiederum, die Möglichkeit, mit der Volksversammlung eine vom Senat unabhängige Politik zu betreiben, zu unterbinden, um damit die Stellung des Senats neu zu untermauern. Andererseits wollte Sulla auch die Ritter als zweitoberste Gesellschaftsschicht neutralisieren, um eine weitere politische Konkurrenzierung des Senatorenstandes zu vermeiden. ${ }^{21}$ Dabei setzte er mit seinen Reformen ein Anliegen durch, das schon M. Livius Drusus als optimatisch gesinnter Volkstribun des Jahres 91 v. Chr. vorgebracht hatte, nämlich die Aufnahme von 300 Rittern in den Senat, der dann die Richter für die Geschworenenhöfe stellt. ${ }^{22}$ Als Diktator war er nun aber in einer stärkeren Position als die Tribunen, deren Möglichkeiten er zusätzlich begrenzt hatte.

Trotz des Hinweises von Livius (per. 89), dass Sulla das Gesetzgebungsrecht der Volkstribunen gänzlich aufgehoben habe, wurde im Jahre 81 v. Chr. möglicherweise nur die Vorschrift über die Vorberatung im Senat erneuert. ${ }^{23}$ Dies stellte in sich schon eine erhebliche Beschneidung des tribunizischen Rogationsrechts dar und kam damit für die popularen Tribunen geradezu einem Gesetzesverbot gleich. $\mathrm{Zu}$ belegen ist jetzt jedenfalls, dass den Tributcomitien die Abstimmungsbefugnis erhalten blieb. Sulla beantragte vor der Tribusversammlung das Gesetz über die Erhöhung der Quaestorenzahl auf zwanzig ${ }^{24}$ und ließ 300 neue Senatoren von ihnen wählen. ${ }^{25}$

Falls die tribunizische lex Antonia de Termessibus noch aus der Zeit vor der Restitution der tribunizischen Rechte im Jahre 70 v. Chr. stammt, wäre das Rogationsrecht gesichert. ${ }^{26}$ Einen Hinweis dafür kann man in der mit dem Gesetz

19 Broughton (1952) 47. 57. 60.

20 Zur Datierung vgl. Lengle (1899) III; Broughton (1952) 74(ff.). Wittmann (1984) 569 geht davon aus, dass die Reformen dem Volk vorgelegt wurden und zwar in Form der Centurien.

21 Dazu Hantos (1988) $54 \mathrm{f}$.

22 Vgl. Gabba (1976); Burckhardt (1988) 256 ff.; Hantos (1988) 50; Thommen (1989) 116. 118. 131. 23 Lange (1876) 852; Herzog (1884) 512; Lengle (1899) 13; Thommen (1989) $131 \mathrm{f}$. Mit einer Verschärfung der Maßnahmen bzw. einem Gesetzesverbot rechnen aufgrund von Livius Lengle (1937) 2485; Seager (1994) 201; Lintott (2005) $140 \mathrm{f}$.

24 Rotondi (1912) 353 f.; Hurlet (1993) 158.

25 App. civ. 1,100,468; vgl. Mommsen (1887/3) 189 Anm. 2; Gabba (1956) 128 ff.; Gabba (1967) 343 ff.: Jede Tribus wählte eine bestimmte Zahl, also acht oder neun Senatoren (anders Drumann [1902] 411).

26 Zur lex Antonia: CIL I² 2, 589 (Dessau, ILS I, 38; Bruns, FIR 14); Broughton (1952) 139 (68 v. Chr.). Mommsen (1887/2) 312 Anm. 1 datierte das Gesetz ins Jahr 71 v. Chr. und betrachtete es wie viele nach ihm als Beleg für das Rogationsrecht unter Sulla. 
verbundenen Formel de s(enatus) s(ententia) erkennen. H. B. Mattingly hat sich aufgrund von prosopographischen und inhaltlichen Gründen zuletzt wieder für das Jahr 72 v. Chr. stark gemacht. ${ }^{27}$ Dabei übergeht er allerdings, dass C. Antonius, nach dem das Gesetz benannt ist, wohl mit C. Antonius Hibrida zu identifizieren ist, der im Jahre $70 \mathrm{v}$. Chr. von der Senatsliste gestrichen wurde ${ }^{28}$ und daraufhin die Güter des Censors Cn. Cornelius Lentulus Clodianus weihte. ${ }^{29}$ Dies dürfte er in seiner späteren Funktion als Volkstribun getan haben, die er damit erst nach dem Jahre 70 v. Chr. ausgeübt hätte. ${ }^{30}$ Entscheidend an der Gesetzgebung Sullas ist jedenfalls, dass die tribunizische Gesetzesinitiative bis zur Aufhebung der Restriktionen im Jahre 70 v. Chr. unter der Kontrolle des Diktators bzw. des Senats blieb. ${ }^{31}$ Dies sieht nach einem Erfolg der sullanischen Politik aus, verdeckt jedoch weitergehende Bedürfnisse, die mit den Einschränkungen angestaut wurden.

Klar bezeugt und unumstritten unter den sullanischen Beschränkungen sind

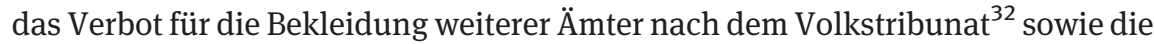
Aufhebung der Volksgerichtsbarkeit. ${ }^{33}$ Das Ämterverbot machte das Volkstribunat für ambitionierte nobiles unattraktiv, so dass in den Jahren des eingeschränkten Tribunats vermehrt Vertreter von unbekannteren oder ins Hinter-

27 Mattingly (1997) (vgl. auch Béranger [1966] 726; Hantos [1988] 78 [Anm. 11]). Ferrary (1985) $439 \mathrm{ff}$. hat inhaltliche Argumente für ein Datum nach 70 v. Chr. vorgebracht (vgl. auch Millar [1998] 77 ff.). Entfallen können die Jahre 71 und 69 v. Chr. sowie wohl auch das Jahr 70 v. Chr., da wir für diese Jahre Tribunen kennen, die in der von allen zehn Mitgliedern des Tribunenkollegiums eingebrachten lex Antonia nicht aufgeführt sind: 71 v. Chr. M. Lollius Palicanus, 70 v. Chr. wohl Plautius, 69 v. Chr. Q. Manlius und Q. Cornificius (vgl. Béranger [1966] 726; Mattingly [1997] 69 Anm. 3). Es anerbieten sich daher nur noch die Jahre 68 und 72 v. Chr., das als terminus post quem feststeht: In lin. 3 der Inschrift werden die Konsuln des Jahres 72 v. Chr. genannt.

28 Niccolini (1934) 249 f.; Broughton (1952) 126 f. 138.

29 Cic. dom. 124; comm. pet. 8; Asc. p. 65 f. St.; vgl. Béranger (1966) 733.

30 Die Weihung war jedenfalls nicht der Grund des Senatssitzverlustes, wie aus Asconius hervorgeht, und dürfte in Analogie zum Fall des C. Atinius Labeo Macerio, Volkstribun 131 v. Chr. (Cic. dom. 123), erst als Reaktion auf den censorischen Akt erfolgt sein (vgl. Thommen [1989] 132 Anm. 15; 188).

31 Für die Zeit der sullanischen Beschränkungen sind auch die lex Antia sumptuaria (Rotondi [1912] 367 f.; Béranger [1966] 733; Mattingly [1997] 68. 71) und die lex Visellia über die cura viarum (Mommsen [1887/2] 668 f.; Rotondi [1912] 367; Syme [1963] 57. 60; Kelly [1970] 134) in Betracht zu ziehen, die L. Volcatius (als Mitautor der lex Antonia) das Amt eines curator viarum ermöglichte. Niccolini (1934) 422 f. datiert die lex Visellia vor 81 v. Chr., Broughton (1952) 132. 136 Anm. 6 in die Jahre 70/69 v. Chr. Zur lex Plautia de reditu Lepidanorum, die möglicherweise ins Jahr 70 v. Chr. gehört (Last [1951] 293. 896 note 3; Broughton [1952] 128. 130 Anm. 4; Hantos [1988] 78f.), vgl. Thommen (1989) 125.

32 App. civ. 1,100,467.

33 Cic. Verr. 1,38. 
treffen geratenen Familien das Amt übernahmen. ${ }^{34}$ Die zweifelhaften Auswirkungen der Verbannung der Tribunen aus der Ämterlaufbahn haben jedenfalls dazu geführt, dass die Bestimmung im Jahre 75 v. Chr. bereits wieder aufgehoben wurde. ${ }^{35}$

Mit dem Verbot der tribunizischen Volksgerichte verhinderte Sulla, dass über die Volksversammlung unliebsame politische Verfolgungen betrieben werden konnten. Solche Volksgerichte waren unter Cinna in den Jahren 87/6 v. Chr. wiederholt gegen die Anhänger Sullas und sogar den Feldherrn selbst eingesetzt worden. ${ }^{36}$ Aufs Ganze gesehen war die Zahl der Volksprozesse in vorsullanischer Zeit jedoch zurückgegangen, da die Geschworenenhöfe offenbar auch für die Tribunen den einfacheren Weg der Anklage boten; mit Sullas systematischem Ausbau der quaestiones stand jetzt ein umfassendes Angebot an ständigen Gerichtshöfen zur Verfügung, so dass das Volksgericht nicht nur vorübergehend, sondern auch nach der Wiederherstellung der tribunizischen Rechte im Jahre 70 v. Chr. fast vollständig zum Erliegen kam. ${ }^{37}$

Bei Cicero (leg. 3,22) ist überliefert, dass Sulla den Volkstribunen die Möglichkeit nahm, Unrecht zu tun, ihnen aber weiterhin gestattete, Hilfe zu leisten. Demnach ist ihnen zumindest das ius auxilii, welches das Interzessionsrecht gegen magistratische Übergriffe beinhaltete, belassen worden. Hierzu sind uns auch konkrete Fälle bekannt. ${ }^{38}$ Das ius auxilii scheint demnach in allen Kreisen unbestritten gewesen zu sein. Es stellte kein unmittelbares Instrument im politischen Tageskampf dar und bildete somit auch keine Gefahr für die res publica. Andererseits gab es dem Volkstribunat eine grundlegende Legitimation, ${ }^{39}$ mit der zugleich weitergehenden Ansprüchen entgegengewirkt werden konnte.

Außerhalb des ius auxilii ist in der Forschung wiederholt mit einem allgemeinen Interzessionsverbot für die Volkstribunen gerechnet worden. ${ }^{40}$ Dagegen spricht zunächst Caesar (civ. 1,5,1. 7,3), der besagt, dass Sulla den Tribunen das Interzessionsrecht belassen habe. ${ }^{41}$ Von Cicero (Verr. 2,1,155) erfahren wir, dass

34 Syme (1963) 55; Thommen (1989) 26; Kondratieff (2003) $88 \mathrm{f}$.

35 S. unten S. 554.

36 S. oben Anm. 17.

37 Thommen (1989) 150 ff. 167. Zu den sullanischen Gerichtshöfen Lengle (1899) 17 ff.; Hantos (1988) $154 \mathrm{ff}$.

38 C. Antonius Hibrida im Jahre 76 v. Chr. (Plut. Caes. 4; Asc. p. 65 St.) und P. Fabius im Jahre 72/ 1 v. Chr. (Cic. Tull. 38 f.); Thommen (1989) 236 f.; Keaveney (2005) 141. 213 Anm. 5.

$39 \mathrm{Vgl}$. Thommen (1988) $362 \mathrm{ff} . ;$ Thommen (1989) 236.

40 Lange (1876) 852; Drumann (1902) 411; Siber (1952) 230; Badian (1955) 110; De Martino (1973) 93; Meyer (1975) 319; Heftner (2006) 216.

41 Lengle (1899) 14 f.; Lengle (1937) 2485 f.; Hantos (1988) 130 ff., bes. 142. Wittmann (1984) 576 Anm. 76 hält die Interzession gegen praetorische und konsularische Gesetze für erlaubt. 
der Tribun Q. Opimius (74 v. Chr.) bestraft wurde, weil er contra legem Corneliam interzediert hatte. Dabei soll sich die Strafe aber gar nicht auf das Veto bezogen haben, sondern sei als Rache dafür gedacht gewesen, dass sich Opimius gegen den nobilis Q. Lutatius Catulus geäußert hatte. ${ }^{42}$ Der Zweck der Interzession bleibt somit unklar. ${ }^{43}$ Sallust (hist. 2,21; ap. Gell. 10,20,10) berichtet weiter, dass der Tribun C. Herennius ex composito gegen Sullas Gesetz, das die Rückkehr des Pompeius aus Afrika regelte, interzedierte. ${ }^{44}$ E. Badian bezieht das Veto aber auf die Abberufung des Prokonsuls Cn. Pompeius Strabo im Jahre 88 v. Chr., ${ }^{45}$ womit die Angelegenheit noch vor die sullanischen Restriktionen $\mathrm{zu}$ liegen käme. Schließlich überliefert Cicero (Cluent. 74), dass der Tribun L. Quinctius im Jahre 74 v. Chr. den Oppianicus-Prozess vertagen ließ, was allerdings als eine spezielle Form des tribunizischen Verbietungsrechts betrachtet werden kann, also nicht zwingend mit einem Veto gegen das magistratische Dekret gleichzusetzen ist. ${ }^{46}$

Auch das Interzessionsrecht im Senat kann für die Jahre nach $81 \mathrm{v}$. Chr. nicht direkt bewiesen werden. ${ }^{47}$ Ein konkreter Hinweis wäre das Veto des Lentulus Spinther, sofern dieser wirklich mit Cn. Cornelius der lex Antonia de Termessibus identisch ist und das Gesetz vor das Jahr 70 v. Chr. gehört. ${ }^{48}$ Da in der Zeit von 133 bis $88 \mathrm{v}$. Chr. keine Interzession im Senat belegt ist und sie daher auch nicht missbräuchlich angewandt worden sein dürfte, ${ }^{49}$ bestand kein Grund, das ius intercedendi auf dieser Ebene einzuschränken. Das Veto war in vorsullanischer Zeit auch sonst nicht gegen den Willen der Senatsmehrheit eingesetzt worden; vielmehr konnte der Senat verschiedentlich von der tribunizischen Interzession profitieren, ${ }^{50}$ so dass es für Sulla wohl gar nicht nötig war, das Vetorecht grundsätzlich zu beschränken. Naheliegender scheint daher, dass Sulla die In-

42 Ps.-Asc. p. 255 St.; Vedaldi Iasbez (1983) 143 f. 158 f. bezeichnet Catulus als das Haupt der konservativen Senatsoligarchie.

43 Als Zielscheibe auszuschließen ist die lex Aurelia de tribunicia potestate, für die sich Q. Opimius selbst einsetzte (vgl. Ps.-Asc. p. 255 St.; Vedaldi Iasbez [1983] bes. 140 Anm. 5, vgl. 142 f. 147; Thommen [1989] 228 Anm. 110); Lintott (1978) 127 glaubt, dass sich die Interzession gegen eine quaestio richtete, die durch ein sullanisches Gesetz eingerichtet worden war.

44 Lengle (1937) 2485; Miltner (1952) 2073; kritisch Hantos (1988) 135 ff. Münzer (1913) $663 \mathrm{f}$. bezweifelt die Übereinkunft, da Herennius auch ein Gegner Sullas gewesen sein kann.

45 Badian (1955) 109 ff.; Badian (1961).

46 Vgl. dazu Mommsen (1887/2) 308 Anm. 1; Lengle (1899) 15; Lefèvre (1910) 104 ff.; Thommen (1989) 229. 231.

47 Mit diesem Recht rechnen Niccolini (1932) 150; Fabbrini (1973) 807; Wittmann (1984) 576; Hantos (1988) 134. Dafür sprechen würde wiederum Caes. civ. 1,5,1. 7,3; anders Lange (1876) 852; Keaveney (2005) 141.

48 Dazu oben Anm. 26.

49 Thommen (1989) 210.

50 Thommen (1989) $216 \mathrm{ff} .228 \mathrm{f}$. 
terzession nur gegen die Anwendung der von ihm erlassenen Gesetze ausschloss, ${ }^{51}$ wie dies auch schon in anderen Fällen verfügt worden war. ${ }^{52}$

Die sullanischen Beschränkungen des Volkstribunats wurden verschiedentlich auch auf das Antrags- und Rederecht im Senat sowie die Einberufung des Senats bezogen. ${ }^{53}$ Dabei wird auf eine Szene bei Appian (civ. 2,29,113) aus dem Jahre 50 v. Chr. verwiesen, in welcher der Tribun C. Scribonius Curio den Senat aufhob, so dass es Pompeius angeblich reute, das Volkstribunat wieder hergestellt zu haben. Dies belegt jedoch nicht, dass die Volkstribunen im sullanischen Senat nicht reden durften und könnte höchstens auf das fehlende Recht, den Senat einzuberufen und aufzuheben, bezogen werden. Falls das Rogationsrecht der Tribunen mit vorheriger Beratung im Senat bestehen blieb, muss das tribunizische Rederecht auch nach dem Jahre $81 \mathrm{v}$. Chr. gewährleistet gewesen sein. Ein Blick auf die tribunizischen Anträge und Äußerungen im Senat zeigt zudem, dass das ius relationis in vorsullanischer Zeit nicht missbraucht worden war. ${ }^{54}$ Sulla dürfte damit keinen Grund gehabt haben, das Rederecht der Tribunen zu unterbinden. Darauf deutet auch Cicero (Cluent. $136 \mathrm{f}$.), der von einem Antrag im Senat im Hinblick auf den iudex quaestionis C. Iunius berichtet, wofür der in diesem Zusammenhang genannte Tribun des Jahres 74 v. Chr., L. Quinctius, in Erwägung zu ziehen ist.

In der Forschung wird davon ausgegangen, dass die Volkstribunen unter Sulla weiterhin contiones einberufen durften. ${ }^{55}$ Das Recht, das Volk zu Informationszwecken in eigenen Versammlungen aufzubieten (ius contionandi), garantierte den Tribunen eine elementare Form des Kontaktes mit der Plebs. Dennoch waren contiones nicht beschlussfähig, so dass keine bindenden Maßnahmen für die res publica getroffen werden konnten. Daher ist es unwahrscheinlich, dass Sulla gegen das ius contionandi einschritt, auch wenn die tribunizischen Ver-

51 Mommsen (1887/2) 308 Anm. 1; Schur (1942) 196; Last (1951) 292; Wittmann (1984) 570; Meier (1997) 255.

52 Lengle (1899) 15; Siber (1952) 230; Hantos (1988) 146; Thommen (1989) 229.

53 Niccolini (1932) $149 \mathrm{f}$.; Lengle (1937) 2485. Valgiglio (1956) $80 \mathrm{ff}$. hält das ius referendi für unwahrscheinlich, die Senatseinberufung für verboten; Fabbrini (1973) 806 möchte ein Antragsverbot aus Cic. Verr. 2,1,155 erschließen, wo Q. Opimius wegen seinen Äußerungen gegen einen nobilis bestraft wird (s. oben Anm. 42), was sich jedoch nicht auf den Senat beziehen muss; Lengle (1899) 14 hatte noch an das Rederecht der Tribunen geglaubt; so auch Lange (1876) 852 und Herzog (1884) 511.

54 Thommen (1989) 195. 201.

55 Mommsen (1887/3) 313 (Anm. 3); Lengle (1899) 13 (Anm. 1); Niccolini (1932) 150; Lengle (1937) 2485 f.; Keaveney (2005) 141. Rubino (1825) 32 ff. hatte noch zwischen contionem habere, contionem dare und in contionem producere unterschieden und bis zum Jahre $75 \mathrm{v}$. Chr. nur das Letztere für zulässig gehalten. 
sammlungen in einigen Fällen für die Nobilität oder einzelne ihrer Mitglieder nachteilig sein konnten. ${ }^{56}$ Dazu kommt, dass die contiones nicht nur eine allgemeine politische Plattform bildeten, sondern insbesondere auch zur Vorbereitung von Gesetzesabstimmungen verwendet wurden. Falls Sulla kein umfassendes Gesetzesverbot für die Tribunen erlassen hat, so muss er zugleich das ius contionandi bestehen lassen haben.

Dennoch soll gemäß Cicero (Cluent. 110) erst L. Quinctius im Jahre 74 v. Chr. wieder die Rostra bestiegen haben. Die Tribunen hatten aber bereits im Jahre $78 \mathrm{v}$. Chr. die Konsuln wieder vor das Volk geführt, ${ }^{57}$ wie es dann auch $\mathrm{Cn}$. Sicinius im Jahre 76 v. Chr. in seiner Agitation für ein uneingeschränktes Volkstribunat tat. ${ }^{58}$ Schon hier zeigt sich, dass Sulla nicht verhindern konnte, dass die contiones gerade auch für die Forderung nach der Restituierung des Volkstribunats rege genutzt wurden und die Restriktionen der tribunizischen Rechte schwerlich dauerhaft aufrechtzuerhalten waren. Die seit dem Ständekampf eingespielten Traditionen ließen sich nicht einfach beiseiteschieben und wurden auch in den Auseinandersetzungen innerhalb der Führungsschicht rege genutzt.

\section{Der Kampf um die Restituierung des Volkstribunats}

Die Beschränkungen des Volkstribunats durch Sulla hatten keinen langen Bestand. Bereits im Jahre $75 \mathrm{v}$. Chr. wurden die Tribunen durch die konsularische lex Aurelia von der Auflage befreit, dass sie nach dem Tribunat keine weiteren Ämter mehr bekleiden durften. ${ }^{59}$ Während des Konsulats von Pompeius und Crassus im Jahre 70 v. Chr. wurden schließlich die restlichen Beschränkungen aufgehoben, so dass die vollen tribunizischen Rechte wieder hergestellt waren. ${ }^{60}$ Im Folgenden soll genauer untersucht werden, wie der Kampf um die tribunicia potestas in den 70er Jahren v. Chr. ablief. Es fragt sich insbesondere, von welcher Seite die Forderung nach dem uneingeschränkten Volkstribunat erhoben wurde und was sich die Beteiligten davon versprachen.

56 Vgl. Thommen (1989) 174 f. Zu den contiones allgemein Hiebel (2009); Tiersch (2009) verweist auf den popularen Einsatz der contiones in der späten Republik und erkennt hinsichtlich des Senats eine „Störung der Kommunikationsbeziehungen zwischen Ober- und Unterschichten“ (64. $67 \mathrm{f}$.$) .$

57 Granius Licinianus 36,33 (in contione).

58 Cic. Brut. 217 (consules produxisset); Thommen (1989) 174. 176.

59 Broughton (1952) 96.

60 Broughton (1952) 126. McDermott (1977) plädiert für eine lex Pompeia. 
Wie bereits angedeutet, blieb den Volkstribunen unter Sulla das ius contionandi erhalten und wurde auch oft in Gebrauch genommen, besonders um die Wiederherstellung der vollen tribunizischen Rechte $\mathrm{zu}$ beantragen. ${ }^{61} \mathrm{Im}$ ersten bezeugten Fall vom Jahre 78 v. Chr. waren die Volkstribunen nach Sullas Ableben übereingekommen, die Restituierung ihrer Rechte $\mathrm{zu}$ fordern; ${ }^{62}$ der Konsul M. Aemilius Lepidus lehnte dies jedoch ab, da dieser Punkt offenbar nicht unter seinen Forderungen figurierte, die einige Änderungen an der sullanischen Gesetzgebung verlangten. ${ }^{63}$ Die Mehrheit der auf der betreffenden contio anwesenden Leute stimmte der Meinung des Lepidus zu, so dass im Volk offenbar noch kein wirkliches Bedürfnis nach Beseitigung der tribunizischen Schranken bestand. Erst im Jahre darauf scheint Lepidus der Wiederherstellung der vollen tribunizischen Rechte concordiae gratia zugestimmt zu haben, wobei in dieser Sache aber keine weitergehende Agitation erkennbar ist. ${ }^{64}$

Im Jahre 76 v. Chr. hat gemäß der Rede des C. Licinius Macer bei Sallust der Volkstribun Cn. Sicinius als erster über die tribunizische Gewalt zu sprechen gewagt ${ }^{65}$ und dabei die Konsuln vergeblich vor die contio geführt. ${ }^{66}$ In seinem heftig betriebenen Kampf ${ }^{67}$ fand er schließlich sogar den Tod. Dies wurde laut Macer vom Volk allerdings nur mit einem effektlosen Murren begleitet. ${ }^{68}$ Breitere Unterstützung dürfte Sicinius in der Öffentlichkeit daher nicht genossen haben.

Im Jahre 75 v. Chr. brachte dann der Konsul C. Aurelius Cotta, der sich im Jahre $91 \mathrm{v}$. Chr. einst selbst um das Volktribunat beworben hatte, ${ }^{69}$ das Gesetz ein, das den Tribunen wieder eine weitere Ämterlaufbahn ermöglichte. Dieses wurde auch von dem Volkstribunen Q. Opimius unterstützt, ${ }^{70}$ der jedoch durch eine Gruppe von Optimaten ausgeschaltet wurde. ${ }^{71}$ Das verdeutlicht, dass der entscheidende Schritt zur Aufwertung des Tribunats schon hier von höherer Seite erfolgen musste. Zudem hatte Opimius seitens der Popularen offenbar keine be-

61 Vgl. Martin (1965) 8 ff.; Gruen (1974) $23 \mathrm{ff}$.

62 S. oben Anm. 57.

63 Flor. 2,11,1 ff. Vgl. Christ (2013) $231 \mathrm{f}$.

64 Sall. hist. 1,77,14. Vgl. Martin (1965) 10 Anm. 7.

65 Sall. hist. 3,48,8, der ihn als L. Sicinius bezeichnet.

66 S. oben Anm. 58.

67 Vgl. Plut. Crass. 7.

68 Sall. hist. 3,48,8.

69 Cic. de orat. 1,25.

70 Ps.-Asc. p. 255 St. Es ist möglich, dass er sich im Senat für das Gesetz geäußert hat, jedoch unwahrscheinlich, dass ihm dies als Interzession gegen die lex Cornelia ausgelegt wurde (vgl. Cic. Verr. 2,1,155), s. oben Anm. 42.

71 Cic. Verr. 2,1,155 berichtet von paucos homines [...] arrogantes. 
sondere Wertschätzung genossen, denn Macer hat ihn in seinem Bericht über die im Kampf um die Restitution bedeutenden Tribunen ausgelassen. ${ }^{72}$

Die lex Aurelia, mit der die erste sullanische Schranke des Volkstribunats fiel, wurde zudem von einem Vertreter der führenden Nobilität eingebracht ${ }^{73}$ und konnte der sullanischen Ordnung auch keinen großen Schaden zufügen. ${ }^{74}$ Da die Kompetenzen der Volkstribunen grundsätzlich unverändert blieben, hatte das aurelische Gesetz auch keine unmittelbaren Auswirkungen auf die Lage der Plebs. ${ }^{75}$ Die lex Aurelia bedeutete damit nur eine Normalisierung im Kampf um die Ämter, der wohl durch die Entwertung des Volkstribunats aus den gewohnten Bahnen geraten war, wobei sich bei den anderen Magistraturen eventuell zusätzliche Engpässe gebildet hatten. ${ }^{76} \mathrm{~J}$. Martin vermutet in dem Gesetz ein Ablenkungsmanöver vom Getreidemangel, der in jenem Jahr herrschte. ${ }^{77} \mathrm{Ob}$ mit der Aufhebung des Ämterverbotes die Unzufriedenheit des Volkes mit dem Ernährungswesen beseitigt werden konnte, ist allerdings fraglich. Die in den Versammlungen anwesende städtische Bevölkerung, die Ch. Meier als plebs contionalis bezeichnet hat, ${ }^{78}$ war bis anhin auch nicht im Kampf gegen die sullanischen Beschränkungen engagiert gewesen. Vielmehr handelte es sich um Leute aus der Oberschicht, die ein uneingeschränktes Volkstribunat forderten - sei es, um die alte Ordnung wiederherzustellen oder ein Instrument für eigenständige Politik in den Händen zu haben.

Im Jahre 74 v. Chr. agitierte als nächster der Volkstribun L. Quinctius für die Restitution des Tribunats. Vorausgegangen war eine Niederlage in einem Giftmordprozess, bei dem sein Mandant offenbar selbst in Korruption verwickelt war. ${ }^{79}$ Aufgrund der Missstände im Gerichtswesen forderte er wieder die vollen tribunizischen Rechte und prozessierte vor einem Geschworenengericht erfolg-

72 Malitz (1972) 385; Vedaldi Iasbez (1983) 146 f. 158 ff. vermutet, dass Opimius zugleich soziale Forderungen in Bezug auf die Agrar- und Italikerfrage stellte, die jedoch nicht die Interessen der plebs urbana betroffen hätten.

73 Cic. Verr. 2,2,174. Umstritten ist die Stellung des Aurelius Cotta in der Nobilität, die Sallust (hist. 3,48,8) mit ex factione media umschreibt. Malitz (1972) meint, dass Cotta trotz einiger Gegner unter den Optimaten (Asc. p. 53 St.) größeren Einfluss ausgeübt haben muss, auch wenn er nicht zur „Nobilität ,mitten aus der factio““ (373) gehört haben dürfte, denn er bedurfte wohl des Senatsmehrs für seine Gesetze. Vgl. auch Vedaldi Iasbez (1983) 152 Anm. 50.

74 Gruen (1974) $27 \mathrm{f}$.

75 Vgl. Vedaldi Iasbez (1983) 149. 153.

76 S. oben Anm. 34.

77 Martin (1965) 12.

78 Meier (1965) 614; Meier (1997) $114 \mathrm{f}$. Zur jüngsten Diskussion darüber Tröster (2013).

79 Broughton (1952) 103. Zur Vermittlerrolle des Richters Staienus vgl. Cic. Cluent. 65. 75 f. 
reich gegen den iudex quaestionis C. Iunius. ${ }^{80}$ Dadurch verdeutlichte Quinctius, dass er selbst nicht in der Lage war, einen Volksprozess durchzuführen, was durchaus als Argument für die Wiederherstellung der tribunizischen Rechte dienen konnte. Gleichzeitig rief er die vormals mit Rittern besetzten Geschworenenhöfe in Erinnerung, die in vorsullanischer Zeit auf Initiative von Volkstribunen eingerichtet worden waren. ${ }^{81}$ Es ist daher $\mathrm{zu}$ vermuten, dass er auch Unterstützung aus ritterlichen Kreisen erhielt, die dem alleinigen Anspruch der Senatoren auf die Gerichte entgegentraten. ${ }^{82}$

Laut Cicero (Cluent. 110) betrat Quinctius als erster seit den sullanischen Beschränkungen wieder die Rostra, was aufgrund der vorangegangenen tribunizischen Vorführungen von Konsuln in den Jahren 78 und 76 v. Chr. allerdings unwahrscheinlich ist. Quinctius hielt jetzt aber täglich Contionen ab (Cluent. 93) und erreichte auch eine gewisse Popularität (110, vgl. 77. 79); dadurch sah sich der Senat zu einem Beschluss im Hinblick auf das Gerichtswesen veranlasst, dessen gesetzliche Verankerung aber versandete und auch vom Volk (populus) aus Mitleid mit dem Sohn des C. Iunius abgelehnt wurde (136 f.). ${ }^{83}$ Den Tribunen selbst soll der Konsul L. Licinius Lucullus nach langem privatem Zureden und öffentlichen Auseinandersetzungen von seiner Agitation abgebracht haben. ${ }^{84}$ Auch Quinctius war es damit trotz der Berücksichtigung der Ritter noch nicht gelungen, genügend Kräfte für die Wiederherstellung der früheren Gerichtsbarkeit bzw. der vollen tribunizischen Rechte zu mobilisieren.

Im Jahre 73 v. Chr. agierte C. Licinius Macer für die Restituierung des Volkstribunats. In seiner von Sallust überlieferten Rede an die Plebs stellt er unter Rückgriff auf die Zeit der Ständekämpfe erstmals das Volkstribunat als Voraussetzung für die libertas des Volkes dar. ${ }^{85} \mathrm{~J}$. Martin sieht darin eine neue Legitimation für das Volkstribunat, die sich durch die sullanischen Restriktionen auf-

80 Vgl. Thommen (1989) 163 f. 166. Nach Ablauf des Tribunats prozessierte Quinctius gegen den Richter C. Fidiculanius Falcula, wobei der erste Anlauf noch erfolglos war (Cic. Cluent. 103 ff.; Asc. p. 219 St.).

81 Thommen (1989) $109 \mathrm{ff}$.

82 Martin (1965) 9.

83 Kelly (1970) 137 ff. vermutet eine von Quinctius gegen C. Iunius beantragte quaestio extraordinaria und deutet dies als Hinweis auf das Rogationsrecht der Tribunen; Ferrary (1985) 441 leitet aus der Information Ciceros, dass die Konsuln für den Gesetzesantrag gefordert gewesen wären, ein tribunizisches Gesetzesverbot ab.

84 Plut. Luc. 5.

85 Martin (1965) 12 ff. 16 Anm. 1; Wirszubski (1967) 64. Stylow (1972) 15 und Robb (2010) 173 f. gehen davon aus, dass dies schon der Konsul M. Aemilius Lepidus in seiner Rede vom Jahre $78 \mathrm{v}$. Chr. getan hatte (Sall. hist. 1,55,23), wobei dort allerdings nur allgemein auf den Ständekampf verwiesen wird. 
drängte, wobei das Tribunat aber schon lange als Teil und Garant der einst erkämpften Freiheit galt. ${ }^{86}$ Macer beschränkte die libertas dabei nicht auf persönliche Freiheit, sondern forderte die Möglichkeit zur politischen Betätigung. Mit ihr wollte er jedoch kaum eine Politisierung des von „Trägheit“ (ignavia) erfassten Volkes erreichen, sondern vermehrte persönliche Macht in der Auseinandersetzung mit dem Senat. ${ }^{87}$ Gemäß J. Martin sollen die Freiheitsrechte des Volkes de facto vom ungehinderten Wirken der Volkstribunen auch gar nicht betroffen gewesen sein, da das Volkstribunat „nicht Exekutivorgan der plebs, sondern Instrument der herrschenden Schicht der Nobilität“ war. ${ }^{88}$ Dennoch deutet das Argument der libertas auch auf den Missstand, dass eigentliche Initiativen im Sinne des Volkes kaum mehr möglich waren. Aus Macers Rede geht zudem hervor, dass die Erwartungen im Hinblick auf die Restituierung des Volkstribunats bereits auf Pompeius lagen (Sall. hist. 3,48,23). Daher kann auch die vom Redner empfohlene Militärdienstverweigerung (3,48,17 f.) keine ernsthafte Taktik dargestellt haben, zumal die plebs urbana nicht die Basis des Heeres bildete. ${ }^{89}$ Die endgültige Befreiung des Tribunats musste also wiederum von anderer Seite vollzogen werden.

Propagandistische Absichten hegte Macer möglicherweise auch mit seiner Anklage gegen Rabirius, der später wegen Beteiligung an der Ermordung des L. Appuleius Saturninus im Jahre 100 v. Chr. gerichtlich verfolgt wurde. Obwohl Macer offiziell ein sacrilegium einklagte, spielte er hier wohl schon auf einen weitergehenden Prozess gegen Rabirius an, der dann im Jahre 63 v. Chr. auf Mord lautete. ${ }^{90}$ Mit seiner wohl harmloseren Anklage machte er wiederum klar, dass er als Tribun unter den sullanischen Maßnahmen nur beschränktes Anklagerecht hatte. Macer erreichte jedoch erneut keine große Popularität und fehlt in PseudoAsconius' Aufzählung derjeniger Tribunen, die sich für die Restitution des Volkstribunats einsetzten. ${ }^{91}$ Trotzdem dürfte er als Politiker und Historiker mit seinem Wirken durchaus Einfluss auf das Bewusstsein von der tribunicia potestas ausgeübt haben, die gerade auch für führende Kreise attraktiv war.

Der letzte Volkstribun im Kampf um die tribunizischen Rechte war der Picener M. Lollius Palicanus im Jahre 71 v. Chr. ${ }^{92}$ Er beschwerte sich in einer contio, dass der Statthalter Verres den C. Sthenius während dessen Aufenthalt in Rom

86 Martin (1965) 12f. 17.

87 Vgl. Wirszubski (1967) 64; Stylow (1972) 15.

88 Martin (1965) 14; vgl. Stylow (1972) 12. $14 \mathrm{f}$.

89 Vgl. Walt (1997) $13 \mathrm{f}$.

90 Martin (1965) 17 Anm. 2.; Thommen (1989) 164.

91 Ps.-Asc. p. 189 St., vgl. Cic. Brut. 238; Martin (1965) 17.

92 Ps.-Asc. p. 189 St.; Sall. hist. 4,43. 
verurteilt hatte, und führte einen von Verres mit der Rute Geschlagenen vor die Volksversammlung, um damit wohl die ungenügenden Interventionsmöglichkeiten der Volkstribunen zu demonstrieren. ${ }^{93}$ Da die Tribunen von der Volksgerichtsbarkeit ausgeschaltet waren, konnten sie offenbar auch nicht als Leiter eines Provokationsprozesses fungieren; damit wurde die tribunicia potestas zugleich mit dem Provokationsrecht als alte plebejische Errungenschaft in Verbindung gebracht, was dem Volkstribunat weitere Legitimation verlieh. ${ }^{94}$ Palicanus setzte sich schließlich mit dem aus dem Sertoriuskrieg zurückgekehrten Pompeius in Verbindung, der das Konsulat anstrebte, und gab ihm Gelegenheit, vor einer contio die Wiederherstellung der vollen tribunizischen Rechte anzukündigen. ${ }^{95}$

Unter dem Druck des Feldherrn und seines Konsulatskollegen Crassus gelang es nun, die Einschränkungen des Tribunats aufzuheben. ${ }^{96}$ Das Volk (populus) soll zu diesem Vorhaben beifällig gemurmelt und erst laut zugestimmt haben, als Pompeius auch Maßnahmen gegen die Ausplünderung der Provinzen und die Korruption in den Gerichten ankündigte. ${ }^{97}$ Die Volkstribunen hatten das verfügbare Volk zwar schon früher in den Kampf um die tribunizischen Rechte hineingezogen, wie es mit L. Quinctius im Jahre 74 v. Chr. zuerst bezeugt ist. ${ }^{98}$ Das in den Versammlungen anwesende Volk hatte aber bis zuletzt keinen besonderen Druck für die Herstellung der unbeschränkten tribunizischen Kompetenzen ausgeübt. Es stimmte offenbar erst überzeugter zu, als das Tribunat mit konkreten politischen Zielen in Zusammenhang gebracht wurde, wie die Zusammensetzung der Gerichte und deren Rechtspraxis, ${ }^{99}$ für die man sich auch von der Wiedereinsetzung der Censoren Besserung versprach. ${ }^{100}$ Die Bevölkerung sah offenbar nur bedingt einen Nutzen in der Restituierung des Volkstribunats und erachtete die Tribunen nicht zwingend als Interessenvertreter breiter Schichten. Zugleich zeigt sich, dass die Tribunen offensichtlich keine dauerhafte Anhän-

93 Cic. Verr. 2,1,122; 2,2,95-100: Auf konsularischen Antrag sollte ein Senatsbeschluss erfolgen, dass die Anklage in absentia gegen Sthenius ungültig sei; die Volkstribunen erklärten, dass sich Sthenius trotz eines anders lautenden Edikts als Verurteilter in Rom aufhalten dürfe; vgl. Münzer (1927) 1391; Thommen (1989) 170.

94 Vgl. Thommen (1989) 164. 236. 252.

95 Ps.-Asc. p. 220 St.; Cic. Verr. 1,45. Pina Polo (2016) 67 f. glaubt, dass Pompeius die contio selber abhielt.

96 Gemäß Suet. Iul. 5 wurde das Vorhaben auch von Caesar als Militärtribun unterstützt.

97 S. oben Anm. 95.

98 Martin (1965) 22, der von „Demagogie“ spricht. C. Aurelius Cotta hatte schon im Jahre davor non consentiente [...] nobilitate magno populi studio (Asc. p. 53 St.) das Ämterverbot aufgehoben. 99 Cic. Verr. 1,44f.

100 Cic. div. in Caec. 8; Martin (1965) 19f.; Thommen (2006) 7. 
gerschaft im Volke hatten. Die Aussage von Ch. Meier, dass „breiteste Schichten“ auf die Wiederherstellung des Volkstribunats drängten, scheint daher deutlich übertrieben. ${ }^{101}$

Zudem hatten der Senat und einzelne Magistrate in den 80er und 70er Jahren v. Chr. einige Reformen in die Hand genommen, wie sie bis anhin eher von den Volkstribunen zu erwarten gewesen waren, darunter der Schuldenerlass im Jahre 86 v. Chr. (lex Valeria de aere alieno) ${ }^{102}$ sowie die Getreidebeschaffung und Landverteilung an Veteranen in den 70er Jahren v. Chr. ${ }^{103}$ Hier war es offenbar möglich, auf einen Einsatz der Tribunen zu verzichten, um bestimmte Maßnahmen umzusetzen. Dennoch reichten die Beschlüsse auf dem Gebiet der Sozialpolitik nicht aus, um den Wunsch nach der vollen tribunicia potestas zu beseitigen, zumal sich noch andere Interessen mit dem Volkstribunat verbanden. Die hergebrachten Möglichkeiten der Volkstribunen waren v. a. auch für Kreise wichtig, die sich gegen die Mehrheit im Senat durchsetzen wollten und eigene Interessen verfolgten.

Neben der plebs urbana erwähnt Pseudo-Asconius auch die Ritter unter denjenigen, die die Wiederherstellung des Volkstribunats wünschten. ${ }^{104}$ Ansonsten treten die Ritter in den Quellen zum Kampf um die uneingeschränkte tribunicia potestas nicht in Erscheinung. Trotzdem müssen sie als ein wesentliches Element in der Auseinandersetzung um das Tribunat betrachtet werden. Die Ritter hatten in vorsullanischer Zeit über die Volkstribunen Einsitz in die Geschworenenhöfe erhalten, womit sie sich politischen Einfluss verschaffen konnten. Die praetorische lex Aurelia des Jahres 70 v. Chr. brachte jetzt einen Kompromiss zustande, der die alleinige Vertretung der Senatoren in den Gerichten beendete und zu je einem Drittel Senatoren, Ritter und Aerartribunen als Richter einsetzte. ${ }^{105}$ Ein weiterer Kampf um die Gerichte erübrigte sich damit auch für die Ritter. Die Publicanen, als einflussreichste Gruppe unter den Rittern, konnten zudem nach dem Jahre $70 \mathrm{v}$. Chr. über tribunizische Aktionen zumindest teilweise nochmals ihre wirtschaftlichen Interessen vertreten. ${ }^{106}$

101 Meier (1997) 140; die angeführte Stelle Cic. leg. 3,26, in der gefragt wird, wie der populus die erkämpfte tribunicia potestas entbehren könne, gibt das kaum her.

102 Rotondi (1912) 347 f.; Martin (1965) 208.

103 Zur Getreideverteilung Marshall - Beness (1987) 363. 368 f.; vgl. die lex frumentaria des M. Aemilius Lepidus, Cos. 78 v. Chr. (Rotondi [1912] 364); zur Landverteilung Gruen (1974) 37.

104 Ps.-Asc. p. 220 St.; Marshall - Beness (1987) 377. Bei Cic. div. in Caec. 8 (vgl. Verr. 1,44) fordert der populus die uneingeschränkte tribunicia potestas, um die alte Gerichtsbarkeit mit ritterlicher Beteiligung zurückzuerhalten.

105 Broughton (1952) 127. Zu den Aerartribunen Bruhns (1980).

106 Thommen (1989) $139 \mathrm{f}$. 
E. S. Gruen hat allerdings davor gewarnt, die Bestrafung der Richter und die Beseitigung der Korruption in den Gerichten als Hauptmotiv für die lex Aurelia des Jahres 70 v. Chr. zu erachten, da nur zwei der 64 Senatsausschlüsse desselben Jahres wegen richterlicher Korruption erfolgten. ${ }^{107}$ Die Korruption in den 70er Jahren v. Chr. lag zudem nicht über dem Durchschnitt der anderen Jahre. ${ }^{108}$ Gruen sieht die Restitution des Tribunats und die Neuordnung der Gerichte eher als Ausgleich innerhalb des Senatorenstandes, der mit dem Ende der äußeren Bedrohung durch Sertorius im Innern Zugeständnisse machen konnte, die für ihn am sullanischen System ohnehin nicht viel änderten und zudem eine Entlastung des Senats mit sich brachten. ${ }^{109}$

Neben den Rittern müssen daher auch andere Interessenten an der tribunicia potestas in Erwägung gezogen werden, zumal sich eine geschlossene Gruppe in der Opposition gegen das sullanische System nicht erkennen lässt. Vorwiegend treten einzelne Volkstribunen in den Vordergrund, die zum einen für die Erweiterung ihrer eigenen Macht sorgen wollten, zum andern aber auch übergeordnete staatspolitische Ziele im Sinne einer ausgeglichenen Verfassung und auch gerichtlichen - Kontrolle der Führungsschicht verfolgten. ${ }^{110}$ Sie stellten sich nie grundsätzlich gegen das Senatsregime ${ }^{111}$ und hatten nachweislich Beziehungen zu nobiles, ${ }^{112}$ die letztlich auch entscheidend waren. Der Kampf um die Wiederherstellung des Volkstribunats darf somit nicht als spezifisch tribunizische Angelegenheit gesehen werden. Er konnte erst mit breiterer Unterstützung und der Initiative aus führenden Kreisen erfolgreich werden.

Mit C. Aurelius Cotta und Pompeius traten letztlich Personen außerhalb des Volkstribunats für die tribunicia potestas ein. Auch in den Reihen des Senatorenstandes müssen etliche Vertreter vom Nutzen des Volkstribunats als Teil des mos maiorum überzeugt gewesen sein, und diese Meinung hat sich zum Schluss offensichtlich durchgesetzt. Die Restitution des Volkstribunats wurde somit nicht der popularen Seite überlassen ${ }^{113}$ und ging ohne Aufruhr über die Bühne, wie auch schon die entsprechende Forderung im Jahre $71 \mathrm{v}$. Chr. ${ }^{114}$ Es ist daher davon

107 Gruen (1974) 30; vgl. Cic. Cluent. 127.

108 Gruen (1974) 33.

109 Gruen (1974) 27 f. 30.35.

$110 \mathrm{Zu}$ dieser Funktion des Volkstribunats Bleicken (1981) bes. 96. 98.

111 Martin (1965) 23.

112 Gruen (1974) 28. Gemäß Marshall - Beness (1978) 378 handelten sie isoliert. Zu L. Quinctius vgl. Cic. Cluent. 110: Atque idem quanto in odio postea suis ipsis fuit per quos in altiorem locum ascenderat!

113 Cic. leg. 3,26; vgl. dagegen die Kritik des Q. Cicero an Pompeius (Cic. leg. 3,22); dazu Thommen (1988) $366 \mathrm{f}$.

114 Gruen (1974) 27. 
auszugehen, dass der Beschluss vom Jahre 70 v. Chr. von einer Mehrheit im Senat gutgeheißen worden war. ${ }^{115}$ Damit verband sich offenbar die Einsicht, dass das uneingeschränkte Volkstribunat grundlegende Vorteile mit sich brachte und letztlich zum Fortbestand der Republik beitrug.

Dennoch darf eine fundamentale Neuerung, die am sullanischen System vorgenommen wurde, nicht übersehen werden. Es wurde jetzt nämlich wieder möglich, mittels Volksversammlung eine gegen die Senatsmehrheit gerichtete Politik zu betreiben. Damit war ein Ventil wieder hergestellt, von dem auch das Volk in Einzelfällen profitieren und somit ruhig gehalten werden konnte. Sulla hatte als Diktator auf die Unterstützung durch die Volkstribunen verzichten können, genauso wie Cinna und Carbo als Konsuln mit militärischer Macht nicht auf sie angewiesen waren. ${ }^{116}$ Nach dem Rücktritt Sullas zeigte sich aber, dass der Senat nicht mehr als geschlossene und konkurrenzlose Einheit bestehen konnte, da der Machtanspruch von Einzelpersönlichkeiten, aber auch der Ritter, zu groß geworden war, als dass dem Druck der Agitation noch auf Dauer hätte widerstanden werden können. Das Interesse an erweiterten politischen Instrumenten und Wirkungsmöglichkeiten war daher aus verschiedener Sicht ungebrochen. Das Volkstribunat stellte dabei nach wie vor auch eine propagandistische Größe im Sinne des mos maiorum dar.

Bemerkenswert ist schließlich, dass Cicero in seinem Staatsentwurf vom Ende der 50er Jahre v. Chr. nochmals ein vollumfängliches Volkstribunat forderte, da er dessen Macht für begrenzt hielt und es nur wenige gefährliche Vertreter gegeben habe. ${ }^{117}$ Bei Caesar zeigte sich kurz darauf, dass er das Tribunat in seinen Kompetenzen gar nicht mehr einschränken musste. Er hatte sich andere Möglichkeiten eröffnet, die Macht der Volkstribunen zu beschneiden und zu einer leeren Hülle verblassen zu lassen, die vorwiegend symbolische Bedeutung hatte; gleichzeitig löste er die tribunicia potestas zumindest teilweise vom Amt ab und teilte sich selbst das Recht auf einen Tribunenstuhl (ius subsellii) und Unverletzlichkeit (sacrosanctitas) zu. ${ }^{118}$ Folgenreicher als die Wiederherstellung des Tribunats hatte sich daher auf längere Sicht das Beispiel von Sullas Marsch auf Rom, seiner Diktatur sowie der ungenügenden Beschränkung der Promagistratur ausgewirkt. Das Volkstribunat war auch mit den restituierten Rechten nur noch bedingt eine eigenständige politische Größe.

115 Martin (1965) 18; Thommen (1989) 132.

116 Martin (1965) 208.

117 Dazu Girardet (1977); Thommen (1988).

118 Thommen (1989) 103 f. $146 \mathrm{f}$. 


\section{Bibliographie}

Badian (1955): E. Badian, The Date of Pompey's First Triumph, Hermes 83, 1955, 107-118.

Badian (1961): E. Badian, Servilius and Pompey's First Triumph, Hermes 89, 1961, 254-256.

Béranger (1966): J. Béranger, La date de la lex Antonia de Termessibus et le tribunat syllanien, in:

R. Chevallier (Hg.), Mélanges André Piganiol, Bd. 2, Paris 1966, 732-737.

Bleicken (1968): J. Bleicken, Das Volkstribunat der klassischen Republik. Studien zu seiner Entwicklung zwischen 287 und 133 v. Chr. (Zetemata 13), München ${ }^{2} 1968$.

Bleicken (1981): J. Bleicken, Das römische Volkstribunat. Versuch einer Analyse seiner politischen Funktion in republikanischer Zeit, Chiron 11, 1981, 87-108.

Bleicken (2004): J. Bleicken, Geschichte der römischen Republik, München ${ }^{6} 2004$.

Broughton (1952): T.R.S. Broughton, The Magistrates of the Roman Republic, Bd. 2, New York 1952 (ND Atlanta 1984).

Bruhns (1980): H. Bruhns, Ein politischer Kompromiss im Jahr 70 v. Chr.: die lex Aurelia iudiciaria, Chiron 10, 1980, 263-272.

Burckhardt (1988): L. A. Burckhardt, Politische Strategien der Optimaten in der späten römischen Republik (Historia Einzelschriften 57), Stuttgart 1988.

Christ (2002): K. Christ, Sulla. Eine römische Karriere, München 2002.

Christ (2013): K. Christ, Krise und Untergang der römischen Republik, Darmstadt ${ }^{8} 2013$.

De Martino (1973): F. De Martino, Storia della costituzione romana, Bd. 3, Napoli ${ }^{2} 1973$.

Drumann (1902): W. Drumann, Geschichte Roms in seinem Übergange von der republikanischen zur monarchischen Verfassung oder Pompeius, Caesar, Cicero und ihre Zeitgenossen nach Geschlechtern und mit genealogischen Tabellen, Bd. 2, hg. v. P. Groebe, Berlin ${ }^{2} 1902$.

Evola Marino (1974): R. Evola Marino, Aspetti della politica interna di Silla, Palermo 1974.

Fabbrini (1973): F. Fabbrini, Tribuni plebis, Novissimo Digesto Italiano 19, Torino 1973, 778-822.

Ferrary (1985): J.-L. Ferrary, La lex Antonia de Termessibus, Athenaeum 63, 1985, 419-457.

Gabba (1956): E. Gabba, Il ceto equestre e il Senato di Silla, Athenaeum 34, 1956, 124-138.

Gabba (1967): E. Gabba, Appiani bellorum civilium liber primus, Firenze ${ }^{2} 1967$.

Gabba (1976): E. Gabba, M. Livius Drusus and Sulla's Reforms, in: E. Gabba, Republican Rome, the Army and the Allies, Berkeley - Los Angeles 1976, 131-141, 250-255.

Girardet (1977): K. M. Girardet, Ciceros Urteil über die Entstehung des Tribunates als Institution der römischen Verfassung (rep. 2,57-59), in: A. Lippold - N. Himmelmann (Hgg.), Bonner Festgabe Johannes Straub (Beihefte der Bonner Jahrbücher 39), Bonn 1977, 179-200.

Gruen (1974): E. S. Gruen, The Last Generation of the Roman Republic, Berkeley u. a. 1974.

Hantos (1988): Th. Hantos, Res publica constituta. Die Verfassung des Dictators Sulla (Hermes Einzelschriften 50), Stuttgart 1988.

Heftner (2006): H. Heftner, Von den Gracchen bis Sulla. Die römische Republik am Scheideweg 133-78 v. Chr., Regensburg 2006.

Herzog (1884): E. von Herzog, Geschichte und System der römischen Staatsverfassung, Bd. 1, Leipzig 1884 (ND Aalen 1965).

Hiebel (2009): D. Hiebel, Rôles institutionnel et politique de la contio sous la République romaine (287-49 av. J.-C.), Paris 2009.

Hurlet (1993): F. Hurlet, La dictature de Sylla: monarchie ou magistrature républicaine? Essai d'histoire constitutionnelle, Bruxelles - Rome (1993).

Katz (1975): B. R. Katz, The First Fruits of Sulla's March, AC 44, 1975, 100-125.

Katz (1976): B. R. Katz, Studies on the Period of Cinna and Sulla, AC 45, 1976, 497-549. 
Keaveney (1983): A. Keaveney, What happened in 88?, Eirene 20, 1983, 53-86.

Keaveney (2005): A. Keaveney, Sulla. The Last Republican, London ${ }^{2} 2005$.

Kelly (1970): D. H. Kelly, Evidence for Legislation by Tribunes 81-70 B.C., in: B. F. Harris (Hg.),

Auckland Classical Essays presented to E. M. Blaiklock, Auckland - Oxford 1970, 133-142.

Kondratieff (2003): E. J. Kondratieff, Popular Power in Action: Tribunes of the Plebs in the Later Republic, Pennsylvania 2003.

Kunkel - Wittmann (1995): W. Kunkel - R. Wittmann, Staatsordnung und Staatspraxis der

Römischen Republik, 2. Abschnitt: Die Magistratur, München 1995.

Laffi (1967): U. Laffi, II mito di Silla, Athenaeum 45, 1967, 177-213, 255-277.

Lange (1876): L. Lange, Römische Alterthümer, Bd. 1, Berlin ${ }^{3} 1876$.

Last (1951): H. Last, Sulla and the Tribunate, in: CAH IX: The Roman Republic 133-44 B.C., 1951, 291-293, 896 n. 3.

Latta (1999): B. Latta, Die Rede des Volkstribunen C. Licinius Macer in den Historien des Sallust (III 48), MAIA 51, 1999, 205-241.

Lefèvre (1910): E. Lefèvre, Du Rôle des Tribuns de la Plèbe en Procédure Civile, Diss. Paris 1910. Lengle (1899): J. Lengle, Untersuchungen über die sullanische Verfassung, Diss. Freiburg i. Br. 1899.

Lengle (1937): J. Lengle, s. v. Tribunus plebis, in: RE VI.A, 1937, 2454-2490.

Lintott (1978): A. W. Lintott, The quaestio de sicariis et venficis and the Latin lex Bantina, Hermes $106,1978,125-138$.

Lovano (2002): M. Lovano, The Age of Cinna. Crucible of Late Republican Rome (Historia Einzelschriften 158), Stuttgart 2002.

Malitz (1972): J. Malitz, C. Aurelius Cotta Cos. 75 und seine Rede in Sallusts Historien, Hermes 100, 1972, 359-386.

Marshall - Beness (1987): B. Marshall - J. L. Beness, Tribunician Agitation and Aristocratic Reaction 80-71 B.C., Athenaeum 65, 1987, 361-378.

Martin (1965): J. Martin, Die Popularen in der Geschichte der Späten Republik, Diss. Freiburg i. Br. 1965 (abgedruckt in: J. Martin, Bedingungen menschlichen Handelns in der Antike. Gesammelte Beiträge zur Historischen Anthropologie, hg. v. W. Schmitz, Stuttgart 2009, 25-195).

Mattingly (1997): H. B. Mattingly, The Date and Significance of the Lex Antonia de Termessibus, Scholia 6, 1997, 68-78.

McDermott (1977): W. C. McDermott, Lex de tribunicia potestate (70 B.C.), CPh 72, 1977, 49-52. Meier (1965): Ch. Meier, s. v. Populares, in: RE Suppl. X, 1965, 549-615.

Meier (1997): Ch. Meier, Res publica amissa. Eine Studie zu Verfassung und Geschichte der späten römischen Republik, Frankfurt a. M. ${ }^{3} 1997$.

Meyer (1898): Ed. Meyer, Die angebliche Centurienreform Sullas, Hermes 33, 1898, 652-654.

Meyer (1975): E. Meyer, Römischer Staat und Staatsgedanke, Zürich - München ${ }^{4} 1975$.

Millar (1998): F. Millar, The Crowd in Rome in the Late Republic, Ann Arbor 1998.

Miltner (1952): F. Miltner, s. v. Cn. Pompeius Magnus, in: RE XXI.2, 1952, 2062-2211.

Mommsen (1887): Th. Mommsen, Römisches Staatsrecht, Bd. 1-3, Leipzig ${ }^{3} 1887$ (ND Basel Stuttgart 1963).

Münzer (1913): F. Münzer, s. v. C. Herennius (7), in: RE VIII, 1913, 663-664.

Münzer (1927): F. Münzer, s. v. M. Lollius Palicanus, in: RE XIII, 1927, 1391.

Niccolini (1932): G. Niccolini, Il tribunato della plebe, Milano 1932.

Niccolini (1934): G. Niccolini, I fast dei tribuni della plebe, Milano 1934. 
Nicolet (1959): C. Nicolet, Note sur Appien, B.C., I, 100, 467: Sylla et la réforme électorale (?), MEFRA 71, 1959, 211-225.

Pina Polo (2016): F. Pina Polo, Magistrates-elect and their potestas contionandi in the Late Roman Republic, Historia 65, 2016, 66-72.

Robb (2010): M. A. Robb, Beyond Populares and Optimates. Political Language in the Late Republic (Historia Einzelschriften 123), Stuttgart 2010.

Rotondi (1912): G. Rotondi, Leges publicae populi romani, Milano 1912 (2. ND Hildesheim 1990). Rubino (1825): J. Rubino, De tribunicia potestate qualis fuerit inde a Sullae dictatura usque ad primum consulatum Pompeji, Diss. Kassel 1825.

Schur (1942): W. Schur, Das Zeitalter des Marius und Sulla (Klio Beiheft 46), Leipzig 1942 (2. ND Aalen 1968).

Seager (1994): R. Seager, Sulla, in: CAH IX, ${ }^{2} 1994$ (ND 2001), 165-207.

Siber (1952): H. Siber, Römisches Verfassungsrecht in geschichtlicher Entwicklung, Lahr 1952. Stylow (1972): A. U. Stylow, Libertas und Liberalitas. Untersuchungen zur innenpolitischen Propaganda der Römer, Diss. München 1972.

Syme (1963): R. Syme, Ten Tribunes, JRS 53, 1963, 55-60.

Thommen (1988): L. Thommen, Das Bild vom Volkstribunat in Ciceros Schrift über die Gesetze, Chiron 18, 1988, 357-375.

Thommen (1989): L. Thommen, Das Volkstribunat der späten römischen Republik (Historia Einzelschriften 59), Stuttgart 1989.

Thommen (2006): L. Thommen, Res publica constituenda. Die Verfassung Sullas und ihre Aufhebung, Gymnasium 113, 2006, 1-13.

Tiersch (2009): C. Tiersch, Politische Öffentlichkeit statt Mitbestimmung? Zur Bedeutung der contiones in der mittleren und späten römischen Republik, Klio 91.1, 2009, 40-68.

Tröster (2013): M. Tröster, Roman Politics and the Whims of the Crowd. The Plebs Contionalis Revisited, Latomus 72, 2013, 128-134.

Valgiglio (1956): E. Valgiglio, Silla e la crisi repubblicana, Firenze 1956.

Vedaldi lasbez (1983): V. Vedaldi lasbez, Un silenzio di Macro (Sall. Hist. 3.48.9-11 M), MEFRA 95, 1983, 139-161.

Walt (1997): S. Walt, Der Historiker C. Licinius Macer. Einleitung, Fragmente, Kommentar, Stuttgart - Leipzig 1997.

Williams (2004): P. Williams, The Roman Tribunate in the ,Era of Quiescence‘ 287-133 BC, Latomus 63, 2004, 281-294.

Wirszubski (1967): Ch. Wirszubski, Libertas als politische Idee im Rom der späten Republik und des frühen Prinzipats, Darmstadt 1967.

Wittmann (1984): R. Wittmann, Res publica recuperata. Grundlagen und Zielsetzung der Alleinherrschaft des L. Cornelius Sulla, in: D. Nörr - D. Simon (Hgg.), Gedächtnisschrift für Wolfgang Kunkel, Frankfurt a. M. 1984, 563-582. 